\title{
Risk Factors for Phlebitis in Patients with Peripheral Intravenous Catheters
}

\author{
Fengmei Tan ${ }^{1}$, Silin Zheng ${ }^{1}$, Hongyan $\mathrm{Wu}^{1}$, Lixia Nie ${ }^{1}$, Wenhua $\mathrm{Li}^{1} \&$ Liping $\mathrm{Li}^{1}$ \\ ${ }^{1}$ Department of Hematology, Affiliated Hospital of Southwest Medical University, Luzhou, Sichuan, China \\ Correspondence: Fengmei Tan, Department of Hematology, Affiliated Hospital of Southwest Medical University, \\ Luzhou, Sichuan 646000, China. Tel: 86-136-796-82678. E-mail: 2490469619@qq.com
}

Received: September 16, 2019 Accepted: Novmber 22, 2019 Online Published: December 5, 2019

doi:10.5539/gjhs.v12n1p41

URL: https://doi.org/10.5539/gjhs.v12n1p41

\begin{abstract}
Introduction: The study investigated the risk factors of phlebitis associated with infusion by peripheral intravenous catheter (PIVC).

Methodology: Hospitalized adults $(n=506)$ were placed with PIVCs by trained nurses. Rates of phlebitis were noted according to PIVC gauge, insertion site, and dwell time; and type and volume of infusion solution.

Results: The development of phlebitis appeared to be significantly associated with the size of the outer diameter of the PIVC. The rate of phlebitis among patients given a hypertonic infusion solution was significantly higher; and also associated with infusion volume. There was no difference in phlebitis development among insertion sites, and dwell times among these sites were comparable.

Conclusion: The risk of phlebitis increased with the PIVC size and volume of infusion solution, and use of a hypertonic solution. The recognition of risk factors and standardized intervention may reduce the occurrence of phlebitis associated with PIVC use.
\end{abstract}

Keywords: Peripheral intravenous catheters; phlebitis; risk factors

\section{Introduction}

The peripheral intravenous catheter (PIVC) is the most common means of vascular access for administering intravenous fluids or parenteral nutrition. It has been reported that $\sim 33-67 \%$ of inpatients are administered treatment via PIVC delivery (Boyd, Aggarwal, Davey, Logan, \& Nathwani, 2011). Because the PIVC is a foreign body placed in the vessel, adverse consequences are possible if used improperly, or after long-term infusion of drugs. Complications include catheter extrusion, occlusion, thrombosis, infection, infiltration, and phlebitis. Such failures result in replacement of the PIVC and interruption in treatment.

The principle reason for PIVC failure is phlebitis, a localized redness, pain along the length of the vein, or a cord-like or hard vein (Enes, Opitz, Faro, \& Pedreira Mde, 2016; Piper et al., 2018), occurring in as many as $41.2 \%$ of patients (Pasalioglu \& Kaya, 2014). Phlebitis can prolong hospitalization, increase medical cost, and overwhelm the workload of the medical staff. In rare cases, phlebitis may lead to serious bloodstream infections that can be threaten life(Hadaway, 2012).

Although many studies have focused on phlebitis associated with a PIVC, there is little information regarding risk factors, which restricts the ability of medical care personnel to prevent or anticipate this complication. Features that have been putatively associated with phlebitis include the infusion site, dwell time (Pasalioglu \& Kaya, 2014) or size of the PIVC; the type and quantity of infusion solutions administered; and the aseptic technique and dressings (Johann, Danski, Vayego, Barbosa, \& Lind, 2016; Rickard et al., 2015). However, conclusions have been inconsistent ("Infusion Nursing Standards of Practice," 2006; O'Grady et al., 2011; Pasalioglu \& Kaya, 2014; Rickard et al., 2015).

To improve the quality of nursing care, the present study investigated the risk factors for phlebitis occurring in patients with PIVC.

\section{Materials and Methods}

The Ethics Committee of the local Hospital approved this case-control study. All subject participants provided informed consent before recruitment. 


\subsection{Participants}

All hospitalized patients older than 18 years who had a peripheral catheter inserted over 24 hours were eligible for inclusion in this study. Informed consent was obtained before the study. Patients with any of the following were excluded: hypertension, heart disease, diabetes, or cognitive impairment; administered chemotherapy; failed PIVC instertion for the first time, or PIVC dwell time $<24$ hours.

\subsection{Study Design}

The enrolled patients were grouped according to the occurrence of phlebitis, i.e., with or without phlebitis. Sample size was calculated according to previous literature. The catheters were removed when the therapy was completed or if any of the following complications occurred: catheter extrusion, occlusion, phlebitis, thrombosis, infection, or infiltration. Patients' demographic data such as age and gender, and the clinical diagnosis were obtained from the electronic medical record system.

PIVCs were inserted by nurses especially trained in intravenous insertion. The PIVCs were protected with standard simple dressings. Data collection was conducted by the specialist nurses. The PIVC was used as long as clinically required and not replaced daily.

The following characteristics were recorded: PIVC gauge, insertion site, and dwell time; type and volume of infusion solutions administered; reason for PIVC withdrawal; and occurrence of phlebitis.

The primary outcome of the study was the occurrence of phlebitis during PIVC use. All patients with a PIVC were checked twice a day by research nurses for signs of phlebitis, such as localized redness, pain along the length of vein, or a cord-like or hard vein. The Infusion Nurses Society (INS) grading standards were used to scale phlebitis (Pasalioglu \& Kaya, 2014). The grade of phlebitis was based on intensity of pain, redness, and venous cord, which was consisted of a 4 -point scale where $0=$ normal, $1=$ mild, $2=$ moderate and $3=$ severe. A score $>3$ was considered evidence of phlebitis. Positive findings of phlebitis were judged by 2 independent individuals.

\subsection{Statistically Analysis}

Data analysis was performed with Statistical Package for the Social Sciences (SPSS) software, version 17.0. Data with a normal distribution was described as mean \pm standard deviation. One-way analysis of variance was used for comparison between groups, and the chi-squared test for categorical variables. $P<0.05$ was considered statistically significant.

\section{Results}

Patients who conformed to the inclusion criteria numbered 506, and 128 developed phlebitis (Table 1). There were no significant differences in age or gender between patients with and without phlebitis.

Table 1. Demographics of patients, with and without phlebitis, $\mathrm{n}(\%)$

\begin{tabular}{llllll}
\hline & & With & Without & $\chi^{2}$ & \multicolumn{2}{c}{0} \\
\hline \multirow{2}{*}{ Gender } & Male & $56(25.9)$ & $160(74.1)$ & 0.079 & 0.779 \\
& Female & $72(24.8)$ & $218(75.2)$ & & \multirow{2}{*}{0.117} \\
\multirow{2}{*}{ Age, y } & $18-40$ & $14(16.5)$ & $71(83.5)$ & 4.296 & \\
& $41-65$ & $80(27.5)$ & $211(72.5)$ & & \\
\hline
\end{tabular}

\subsection{PIVC Characteristics}

\subsubsection{Dwell Time}

At a PIVC dwell time $<48$ hours, the rate of phlebitis was $42.1 \%$, which was significantly higher than that of the other dwell time categories (Table 2). The rate of phlebitis appeared to decrease with dwell time from 48 to 168 hours, but the rate of $7.4 \%$ at dwell time $145-168$ hours increased to $15.4 \%$ at $>168$ hours.

\subsubsection{PIVC Gauge}

The development of phlebitis appeared to be associated with the size of the outer diameter (od) of the PIVC, that is, inversely associated with the gauge of the PIVC (Table 2$)$. At the smallest outer diameter ( $0.599 \mathrm{~mm}$ od; gauge 24) the rate of phlebitis was $18.9 \%$, which was significantly less than that of the PIVC gauges $22(0.711 \mathrm{~mm}$ od, rate $32.0 \%)$ and $20(0.902 \mathrm{~mm}$ od, rate $35.0 \%)$

\subsubsection{Insertion Site}

In this study, the PIVC insertion sites were the arm, forearm, and wrist (Table 2). These sites were statistically 
comparable with regard to the rate of phlebitis development.

Table 2. PIVC and infusion characteristics of patients, with and without phlebitis, $\mathrm{n}(\%)$

\begin{tabular}{|c|c|c|c|c|c|}
\hline & & With & Without & $\chi^{2}$ & $P$ \\
\hline \multirow{7}{*}{ PIVC dwell time, $\mathrm{h}$} & $<48$ & $61(42.1)$ & 84 (57.9) & \multirow{7}{*}{38.411} & \multirow{7}{*}{0.001} \\
\hline & $49-72$ & $27(27.6)$ & $71(72.4)$ & & \\
\hline & $73-96$ & $18(19.1)$ & $76(80.9)$ & & \\
\hline & $97-120$ & $12(16.2)$ & $62(83.8)$ & & \\
\hline & $121-144$ & $4(9.5)$ & $38(90.5)$ & & \\
\hline & $145-168$ & $2(7.4)$ & $25(92.6)$ & & \\
\hline & $>168$ & $4(15.4)$ & $22(84.6)$ & & \\
\hline \multirow{3}{*}{ PIVC size, gauge } & 24 & $53(18.9)$ & $228(81.1)$ & \multirow{3}{*}{14.112} & \multirow{3}{*}{0.001} \\
\hline & 22 & $39(32.0)$ & $83(68.0)$ & & \\
\hline & 20 & $36(35.0)$ & $67(65.0)$ & & \\
\hline \multirow{3}{*}{ PIVC insertion site } & Arm & $72(24.2)$ & $226(75.8)$ & \multirow{3}{*}{4.140} & \multirow{3}{*}{0.126} \\
\hline & Wrist & $17(20.0)$ & $68(80.0)$ & & \\
\hline & Forearm & $39(31.7)$ & $84(68.3)$ & & \\
\hline \multirow{2}{*}{ Infusion solution type } & Hypertonic & $21(44.7)$ & $26(55.3)$ & \multirow{2}{*}{10.302} & \multirow{2}{*}{0.001} \\
\hline & Isosmotic & $107(23.3)$ & $352(76.7)$ & & \\
\hline \multirow{4}{*}{ Infusion volume/d, $\mathrm{mL}$} & $<500$ & $35(18.6)$ & $153(81.4)$ & \multirow{4}{*}{9.599} & \multirow{4}{*}{0.022} \\
\hline & $500-1000$ & $61(26.9)$ & $166(73.1)$ & & \\
\hline & $1000-1500$ & $21(33.9)$ & $41(66.1)$ & & \\
\hline & $>1500$ & $11(37.9)$ & $18(62.1)$ & & \\
\hline
\end{tabular}

\subsubsection{PIVC Insertion site and Dwell Time}

The PIVC dwell times decreased from insertion in the arm, to forearm, to wrist (Table 3), but the differences did not reach statistical significance.

\subsection{Infusion Characteristics}

\subsubsection{Properties of Infusion Solutions}

The rate of phlebitis among patients given a hypertonic infusion solution (44.7\%) was significantly higher than that of patients who received an isosmotic solution (23.3\%; Table 2)

\subsubsection{Volume of Infusion Solutions}

The rate of phlebitis was significantly associated with infusion volume, from $18.6 \%$ at a volume $<500 \mathrm{~mL}$, to $37.9 \%$ at $>1500 \mathrm{~mL}$ (Table 2).

Table 3. PIVC dwell time at different PIVC insertion sites in patients with phlebitis

\begin{tabular}{lllll}
\hline & Cases, $n$ & PIVC dwell time, $\mathrm{h}$ & $\mathrm{F}$ & $P$ \\
\hline Arm & 296 & $97.92 \pm 58.36$ & 1.200 & 0.303 \\
Forearm & 87 & $87.65 \pm 46.55$ & & \\
Wrist & 123 & $58.36 \pm 60.82$ & & \\
\hline
\end{tabular}

\section{Discussion}

To improve the quality of nursing care for patients for whom therapy is delivered via PIVC, this study investigated the risk factors for phlebitis associated with PIVC. It was determined that among 506 hospitalized adults with a PIVC, 128 developed phlebitis. The rate of phlebitis was significantly higher at a PIVC dwell time <48 hours, compared with the other categories of dwell time, including $>168$ hours. The development of phlebitis appeared to be significantly associated with the size of the PIVC and volume of infusion solution, and the percentage of 
patients with phlebitis given a hypertonic infusion solution was significantly higher than that of patients who received an isosmotic solution. The insertion site had no effect on the rate of phlebitis.

The rate of phlebitis was $25.3 \%$ in our study, which was lower than that reported by Tertuliano et al.(Tertuliano, Borges, Fortunato, Oliveira, \& Poveda, 2014). In the present study, the high incidence of phlebitis in patients with a dwell time $<48$ hours $(42.1 \%)$ may be due to the acute inflammation that generally occurs 24-48 hours after injury (Sieggreen, 2005). However, the rate of phlebitis at a dwell time $>168$ hours was higher than that of a dwell time of 145-168 hours, which may be related to an increased risk of contamination with changes of the PIVC over time. It is also possible that the PIVC can aggravate venous vascular endothelium injury when the dwell time exceeds 168 hours. Therefore, the suggested retention time of a PIVC should be $<168$ hours, and PIVCs should be removed or replaced even when there are no signs of phlebitis. A systematic review found no evidence to support changing catheters every 72 to 96 hours (Webster, Osborne, Rickard, \& New, 2015). Consequently, healthcare organizations may consider changing to a policy whereby catheters are changed only if clinically indicated (Webster et al., 2015). This requires further studies to confirm the optimal timepoint, as well as how changes in PIVC maintenance may improve function.

Previous studies have shown that the insertion/infusion site affects the risk of PIVC failure (Dillon et al., 2008; Tagalakis, Kahn, Libman, \& Blostein, 2002). This should be considered by medical care personnel. The forearm has recently been recommended as the preferred insertion site in the guidelines (Gorski, 2017; O'Grady et al., 2011). In the present study, the PIVC dwell time was longest for PVCs placed in the arm, and shortest for those in the wrist, although the difference did not reach significance. A reason for the lower rate of phlebitis at the wrist may be that the radial vein has a larger diameter. This is in accord with Urbanetto et al. (Urbanetto et al., 2017). To reduce the risk of phlebitis, the insertion/infusion should be inspected at each shift change and the catheter removed if signs of inflammation, infiltration, or blockage are present.

In the present study, the rate of phlebitis associated with a size 22 gauge PIVC was significantly lower than that of the size 20 gauge. This may be because there is less mechanical stimulus of the blood vessel endothelium caused by the smaller PIVC.

The incidence and severity of phlebitis has been found to correlate positively with the volume of infusion solution administered (Uslusoy \& Mete, 2008). In the current study, the rate of phlebitis was highest when a volume of $>1500 \mathrm{~mL}$ infusion solution was administered per day. This may be because the higher lateral pressure of the high volume may lead to mechanical damage to the vessel walls.

Compared with the infusion of an isosmotic fluid, the rate of phlebitis was significantly higher when a hypertonic fluid was used. This is consistent with results reported by Uslusoy et al.(Uslusoy \& Mete, 2008), and may be related to denaturation of vascular endothelial cells caused by increased plasma osmotic pressure, leading to fluid extravasation and hardening of the blood vessels. However, the underlying mechanisms need further elucidation. It is suggested that special care should be taken when applying either a hypertonic or large quantity of infusion fluid.

\section{Conclusion}

The current study is limited, in that investigators were not blinded to the study design, so potential bias may exist regarding outcome assessment. In addition, the study was conducted at a single medical center, so conclusions may be interpreted with caution.

In conclusion, the greatest risk of phlebitis associated with use of a PIVC occurs within 48 hours after placement. Other risk factors include use of a hypertonic fluid, or administration of a high daily volume. Avoiding of preventable risk factors is needed for prevention of phlebitis. Under these conditions, medical personnel should take special care in order to reduce the occurrence of phlebitis. It may be recommended to assess phlebitis with high risk and to take initiative measures specifically for preventing the development of phlebitis.

\section{Acknowledgements}

None.

\section{Competing Interests Statement}

The authors declare that there are no competing or potential conflicts of interest.

\section{References}

Boyd, S., Aggarwal, I., Davey, P., Logan, M., \& Nathwani, D. (2011). Peripheral intravenous catheters: the road to quality improvement and safer patient care. $J$ Hosp Infect, 77(1), 37-41. https://doi.org/10.1016/j.jhin.2010.09.011 
Dillon, M. F., Curran, J., Martos, R., Walsh, C., Walsh, J., Al-Azawi, D., . . O'Shea, D. (2008). Factors that affect longevity of intravenous cannulas: a prospective study. Qjm, 101(9), 731-735. https://doi.org/10.1093/qjmed/hcn078

Enes, S. M., Opitz, S. P., Faro, A. R., \& Pedreira Mde, L. (2016). Phlebitis associated with peripheral intravenous catheters in adults admitted to hospital in the Western Brazilian Amazon. Rev Esc Enferm USP, 50(2), 263-271. https://doi.org/10.1590/S0080-623420160000200012

Gorski, L. A. (2017). The 2016 Infusion Therapy Standards of Practice. Home Healthc Now, 35(1), 10-18. https://doi.org/10.1097/NHH.000000000000481

Hadaway, L. (2012). Short peripheral intravenous catheters and infections. J Infus Nurs, 35(4), 230-240. https://doi.org/10.1097/NAN.0b013e31825af099

Infusion Nursing Standards of Practice. (2006). $J$ Infus Nurs, 29(1 Suppl), S1-92. https://doi.org/10.1097/00129804-200601001-00001

Johann, D. A., Danski, M. T., Vayego, S. A., Barbosa, D. A., \& Lind, J. (2016). Risk factors for complications in peripheral intravenous catheters in adults: secondary analysis of a randomized controlled trial. Rev Lat Am Enfermagem, 24, e2833. https://doi.org/10.1590/1518-8345.1457.2833

O'Grady, N. P., Alexander, M., Burns, L. A., Dellinger, E. P., Garland, J., Heard, S. O., . . Saint, S. (2011). Guidelines for the prevention of intravascular catheter-related infections. Am J Infect Control, 39(4 Suppl 1), S1-34. https://doi.org/10.1016/j.ajic.2011.01.003

Pasalioglu, K. B., \& Kaya, H. (2014). Catheter indwell time and phlebitis development during peripheral intravenous catheter administration. Pak J Med Sci, 30(4), 725-730. https://doi.org/10.12669/pjms.304.5067

Piper, R., Carr, P. J., Kelsey, L. J., Bulmer, A. C., Keogh, S., \& Doyle, B. J. (2018). The mechanistic causes of peripheral intravenous catheter failure based on a parametric computational study. Sci Rep, 8(1), 3441. https://doi.org/10.1038/s41598-018-21617-1

Rickard, C. M., Marsh, N. M., Webster, J., Gavin, N. C., McGrail, M. R., Larsen, E., . . Playford, E. G. (2015). Intravascular device administration sets: replacement after standard versus prolonged use in hospitalised patients-a study protocol for a randomised controlled trial (The RSVP Trial). BMJ Open, 5(2), e007257. https://doi.org/10.1136/bmjopen-2014-007257

Sieggreen, M. (2005). Venous disorders: overview of current practice. J Vasc Nurs, 23(1), 33-35. https://doi.org/10.1016/j.jvn.2004.10.003

Tagalakis, V., Kahn, S. R., Libman, M., \& Blostein, M. (2002). The epidemiology of peripheral vein infusion thrombophlebitis: a critical review. Am J Med, 113(2), 146-151. https://doi.org/10.1016/S0002-9343(02)01163-4

Tertuliano, A. C., Borges, J. L. d. S., Fortunato, R. A. S., Oliveira, A. L., \& Poveda, V. d. B. (2014). Phlebitis associated with peripheral intravenous catheter among in-patients of a Hospital in Vale do Paraíba. REME: Revista Mineira de Enfermagem, 18(2). https://doi.org/10.5935/1415-2762.20140026

Urbanetto, J. S., Muniz, F. O. M., Silva, R. M. D., Freitas, A. P. C., Oliveira, A. P. R., \& Santos, J. (2017). Incidence of phlebitis and post-infusion phlebitis in hospitalised adults. Rev Gaucha Enferm, 38(2), e58793. https://doi.org/10.1590/1983-1447.2017.02.58793

Uslusoy, E., \& Mete, S. (2008). Predisposing factors to phlebitis in patients with peripheral intravenous catheters:

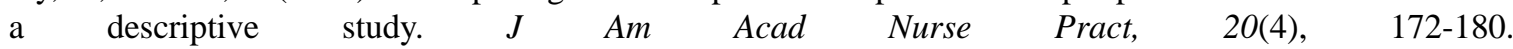
https://doi.org/10.1111/j.1745-7599.2008.00305.x

Webster, J., Osborne, S., Rickard, C. M., \& New, K. (2015). Clinically-indicated replacement versus routine replacement of peripheral venous catheters. Cochrane Database Syst Rev, (8), CD007798. https://doi.org/10.1002/14651858.CD007798.pub4

\section{Copyrights}

Copyright for this article is retained by the author(s), with first publication rights granted to the journal.

This is an open-access article distributed under the terms and conditions of the Creative Commons Attribution license (http://creativecommons.org/licenses/by/4.0/). 